\title{
Linear Late Gadolinium Enhancement In The Basal Anterior Septum And Lateral Wall May Represent The Anterior Septal Perforator Artery And Circumflex Artery, Respectively: A CMR And CCTA Comparison Study
}

\section{Peisong Ma}

Army Medical University (Third Military Medical University)

\section{Yongnning Shang}

Army Medical University (Third Military Medical University)

Yurou Hu

Army Medical University (Third Military Medical University)

Juan Liu

Army Medical University (Third Military Medical University)

Xiaoyue Zhou

Army Medical University (Third Military Medical University)

Jian Wang ( $\square$ wangjian_811@yahoo.com )

Army Medical University (Third Military Medical University) https://orcid.org/0000-0003-1210-0837

\section{Research Article}

Keywords: Anterior septal perforator artery, circumflex artery, linear late gadolinium enhancement, Cardiac magnetic resonance

Posted Date: May 5th, 2021

DOI: https://doi.org/10.21203/rs.3.rs-472315/v1

License: (c) (i) This work is licensed under a Creative Commons Attribution 4.0 International License.

Read Full License 


\section{Abstract}

Purpose: The purpose of this paper was to verify that the linear high-intensity signal on late gadolinium enhancement-cardiac magnetic resonance (LGE-CMR) may represent the contrast enhancement of vessels rather than scars or fibrosis, and to assess whether this linear high-intensity signal will affect the quantification of myocardial fibrosis in patients with hypertrophic cardiomyopathy (HCM).

Methods: A total of 58 patients who underwent both coronary computed tomography angiography (CCTA) and LGE-CMR in our hospital were ultimately enrolled. The definitions of positive linear LGE (LLGE+) were as follows: (1) LLGE in the basal anterior septum or lateral wall, and (2) LLGE observable at $10 \mathrm{~mm}$ or more. All other patients were regarded as negative LLGE (LLGE-). In LLGE+ patients, the length of the LLGE located in the anterior septum and lateral wall was compared with the length of the septal perforator artery and the circumflex artery on CCTA, respectively. For nine patients with HCM, the LGE\% was measured before and after removal of LLGE.

Results: Among the 58 patients, 40 of whom showed LLGE+ and 18 of whom showed LLGE-. For patients with LLGE in the anterior septum, there was a strong correlation between LLGE and anterior septal perforator arteries in length $(r=0.887, p<0.001)$. For patients with LLGE in the lateral wall, LLGE also correlated well with the circumflex arteries in length $(r=0.962, p<0.001)$. In nine patients with $\mathrm{HCM}$, the LGE\% decreased significantly after the removal of LLGE (18.6 [14.15 - 27.5] vs. 12.4 [9.8 - 17.5], $p<0.05)$.

Conclusions: The LLGE in the anterior septum and lateral wall may represent contrast enhancement of the anterior septal perforator artery and the circumflex artery, respectively. This LLGE may overestimate the extent of myocardial fibrosis in patients with hypertrophic cardiomyopathy.

\section{Introduction}

Late gadolinium enhancement-cardiac magnetic resonance (LGE-CMR) can reflect tissue inflammation, infarction and fibrosis, or identify ischemic or non-ischemic cardiomyopathy and be clinically valuable in assessing the prognostic outcome of cardiomyopathy[1-3]. Impaired local outflow of contrast in inactive myocardial tissue results in the accumulation of gadolinium ions, and the shortening of T1 subsequently causes infarcted or fibrotic myocardial tissue to exhibit high signal on LGE, unlike normal myocardial tissue[4-6]. The phase sensitive inversion recovery (PSIR) technique is now widely used for LGE imaging, and it can reduce the operator's dependence on TI values and yield better contrast[7,8].

Different LGE patterns reflect distinct myocardial lesions. For example, LGE that occurs under the epicardium in the lateral wall is often considered to represent myocarditis or sarcoidosis(2). Linear LGE in the mid-wall of the septum always occurs in dilated cardiomyopathy (DCM) [9], and flocculent or lamellar LGE in the basal septum always occurs in hypertrophic cardiomyopathy (HCM)[10]. Therefore, it is important to correctly distinguish the pattern and location of LGE. 
Recent studies suggested that LGE in the basal septum may represent an artery or vein. Nakamura et al. [11] reported that a linear high-intensity lesion in the basal anterior septum could be observed in the shortaxis view on LGE. Compared to the maximum intensity projection (MIP) images of coronary computed tomography angiography (CCTA), the linear high-intensity signal may represent the anterior septal perforator artery. In addition, Masafumi et al. [1] reported that the basal septal perforator vein showed linear high-intensity enhancement in the delayed phase of coronary CT. However, the exact incidence of this phenomenon is unclear and the possibility that the linear LGE (LLGE) of the lateral wall may also be vascular has not been reported.

Therefore, the purpose of this paper was to verify that the LLGE may represent the anterior septal perforator artery in the basal anterior septum or circumflex artery in the lateral wall, and to assess whether this linear high-intensity signal will affect the quantification of myocardial fibrosis in patients with hypertrophic cardiomyopathy (HCM).

\section{Material And Methods}

\section{Study patients}

A total of 61 patients who underwent both CCTA and LGE-CMR between January 2015 and December 2020 in our hospital were retrospectively enrolled. The exclusion criteria included: (1) high-intensity lesion in the anterior septum and lateral wall on LGE images that affects the observation of LLGE due to primary disease, or (2) poor LGE image quality on CCTA. Based on these criteria, three patients were excluded from the study, two of whom had obvious enhancement on LGE imaging (one with HCM and the other with cardiac amyloidosis) (Supplementary Fig. 1) and one who produced poor CMR image quality due to motion artifacts during image acquisition. Therefore, a total of 58 participants were included, of which forty patients ( 34 males, $45 \pm 15$ years of age) with LLGE + and eighteen patients ( 13 males, $45 \pm 17$ years of age) with LLGE- were studied. The study was approved by the Institutional Review Board of our hospital. This study was designed as a retrospective chart review, so written patient consent was waived.

\section{CMR protocols}

Subjects were scanned with on 3-Tesla MRI scanner (Trio, Siemens Healthineers, Erlangen, Germany) using a 12-channel body array coil. Before scanning, the patient-specific CV shimming sequence was used to shimmer the heart region to improve field uniformity. A breath-hold, retrospective, electrocardiogram (ECG)-gated balanced steady state free-precision (bSSFP) cine sequence was used to obtain cine images, including the LV 2-and 4-chamber long-axis and short-axis stack covering the entire LV from base to apex. LGE images were acquired 10 minutes after injection of a $0.2 \mathrm{mmol} / \mathrm{kg}$ bolus of gadoteric acid meglumine (Dotarem, Guerbet, BP7400, F95943, Roissy CdG Cedex, France), with a Phase

Sensitive Inversion Recovery (PSIR) sequence with the following parameters: FOV $400 \times 300 \mathrm{~mm}^{2}$, matrix $256 \times 166$, TR/TE $301.7 / 1.09 \mathrm{~ms}$, flip angle 35 degrees, $6 \mathrm{~mm}$ thickness, and slices including the LV 2and 4-chamber long-axis and short-axis stack covering the entire LV from base to apex. 


\section{CCTA protocol}

The CCTA was conducted using a 64-multidetector row scanner (SIEMENS; SOMETOM Definition, Germany). Metoprolol was used at $5 \mathrm{mg}$ increments up to a total possible dose of $25 \mathrm{mg}$ to reduce the heart rate if it was greater than 75 beats per minutes in the absence of any contraindications. A total of 40-50 ml of iohexol (350mg iodine/ml; Yangtze River Pharmaceutical Co., Ltd, Jiangsu) was injected for angiography into an antecubital vein at a flow rate of $5 \mathrm{ml} / \mathrm{s}$ followed by a $50 \mathrm{ml}$ flush of normal saline at the same flow rate. The scan parameters were as follows: matrix, $512 \times 512$; collimation, $64 \mathrm{~mm} \times 0.625$ $\mathrm{mm}$; tube rotation time, $350 \mathrm{~ms}$; and tube current, 300-400 mA at 100-120 kV for subjects based on their body size. Retrospective ECG-gated mode (heart rate $>60$ beats/min) or prospective ECG-gated mode (heart rate $\leq 60$ beats $/ \mathrm{min}$ ).

\section{Image analysis}

Short-axis LGE images were analyzed by two radiologists with more than ten years of experience and who were blinded for clinical events. The definitions of LLGE + were as follows: (1) In the anterior septum or lateral wall, the linear high-intensity signal could be clearly seen in the epicardium or mid-wall of the myocardium, and (2) LLGE observable at $10 \mathrm{~mm}$ or more in the anterior septum and lateral wall. Based on previous studies, $15 \mathrm{~mm}$ was set as the cut-off value to exclude the possibility of artifacts[11]. In this study, LGE images were obtained by the PSIR technique with higher resolution, so $10 \mathrm{~mm}$ was used as the criterion for determining LLGE. Other patients were regarded as LLGE-. In addition, the length of LLGE in the anterior septum and lateral wall was measured.

For nine patients with hypertrophic cardiomyopathy (HCM), LGE quantification was measured using CVI42 software (Circle Cardiovascular Imaging Inc., Calgary, Alberta, Canada). LV endocardial and epicardial borders on the LGE images were manually traced, taking care to avoid partial-volume effects from the blood pool and epicardial fat. LGE was defined as the signal intensity of the myocardium greater than or equal to 5 standard deviations above that of the remote myocardium, which has been shown to have the best accuracy with histology-verified fibrosis in HCM[12]. LGE\% was used to express the percentage of enhancement area mass in the total mass of the left ventricular myocardium. After manually removing the LLGE region, the LGE\% was measured again.

The short-axis MIP images of the left ventricle were reconstructed on the diastolic images of the CCTA, and the slices corresponded to the LGE, with a slice thickness of $8 \mathrm{~mm}$. The CCTA images were evaluated by another radiologist to determine whether there was a septal perforator artery or circumflex branch in the epicardium or mid-wall of the myocardium, and the lengths of the septal perforator artery and circumflex branch artery were measured. All the CCTA images were evaluated on a 3D image analysis workstation.

\section{Statistical analysis}


The Kolmogorov-Smirnov test was used to test the normality of the variables. Quantitative data with a normal distribution were expressed as the mean and standard deviation (SD), whereas the other measurement data were expressed as the median (first quartile, third quartile). The counting data were showed as the number of cases. Independent Student's t tests were used to compare the differences in the continuous data, and the chi-squared test or Fisher's exact test were used to evaluate the differences in proportions. For LLGE + patients, Pearson correlation coefficients were used to assess the correlation between LLGE length on CMR and the artery on CCTA. The LGE\% between the original measurement and second measurement after removing the LLGE manually was compared using the paired $t$ test among the HCM patients. Bland-Altman analysis was used to evaluate the consistency of lengths between LLGE and the artery on CCTA. $P<0.05$ was considered to be statistically significant. Data were analyzed using SPSS (version 21.0, Statistical Package for the Social Sciences, International Business Machines, Inc., Armonk, New York, USA) and GraphPad Prism (version 6.01, GraphPad Software, Inc., La Jolla, California, USA).

\section{Results}

The clinical characteristics of the patients are shown in Table 1. The proportion of patients with hypertension was significantly higher in the LLGE- patients than in the LLGE + patients $(p<0.001)$. There were no significant differences between the two groups in age, gender, LVEF, LVEDV, or LVESV (all $P>$ 0.05).

In the anterior septum, the anterior septal perforator artery on CCTA was similar on LLGE in shape and running direction (Fig. 1, Table 2). A total of 35 patients (35/58,60.3\%) showed LLGE, and anterior septal perforator arteries were all observed in the same region on CCTA $(100.0 \%)$. The length of the LLGE in the anterior septum correlated well with that of the septal perforator arteries on the MIP images on CCTA ( $r=$ $0.887, p<0.001$, Fig. 2). In contrast, of the 54 CCTA + patients, LLGE were not identified in 19 of them on LGE. The measured length of the anterior septal perforator arteries was significantly shorter in the LLGEpatients ( $19.8 \pm 5.5 \mathrm{~mm}$ vs. $30.7 \pm 8.9 \mathrm{~mm}, \mathrm{p}<0.001$, Fig. 3 ). Among the patients showing LLGE in the anterior septum, $27(27 / 35,77.1 \%)$ had LLGE traveling sub-epicardially, and $8(8 / 35,22.8 \%)$ had LLGE traveling intramyocardially (Table 3).

A similar LLGE was found in the lateral wall on short-axis views of the LGE. Of the 58 patients, $10(10 / 58$, $17.2 \%$ ) showed LLGE in the lateral wall, a number that was lower than the proportion of LLGE found in the anterior septum ( 16.9 vs. $60.3 \%, p<0.001)$. Circumflex branch arteries were all observed in the same region on CCTA (100.0\%), similar in shape and running direction to the LLGE (Fig. 4, Table 4). LLGE also correlated well with the circumflex branch arteries in terms of length $(r=0.962, p<0.001$, Fig. 5$)$. There were 4 patients $(4 / 58,6.8 \%)$ with visible circumflex branch arteries on CCTA but no LLGE in the lateral wall. Of the patients exhibiting LLGE in the lateral wall, 8 (8/10, 80.0\%) with LLGE were located subepicardially, and the other $2(2 / 10,20.0 \%)$ were located intramyocardially (Table 3 ). 
By analyzing LGE images of 9 patients with $\mathrm{HCM}$ and measuring LGE\% before and after removal of LLGE, there was a significant difference between the two measurements (18.6, [14.15-27.5] vs. 12.4, [9.8-17.5] $p<0.05$ ), with a significant decrease in LGE\% after removal of LLGE (Fig. 6).

\section{Discussion}

LGE-CMR is the "gold standard" for noninvasive evaluation of myocardial fibrosis[5]. However, it is important to note that not all high-intensity enhancement on LGE images represent scarring or fibrosis. In the present study, LLGE located in the lateral wall of the CMR may represent a circumflex branch artery that travels within the myocardium, and LLGE located in the anterior septum may represent an anterior septal perforator artery. This "false lesion" may exaggerate the actual degree of myocardial fibrosis in patients with HCM.

The LLGE in the anterior septum was noted to be similar to the anterior septal perforator artery on coronary CT in its shape and running, and the ratio of LLGE (60.3\%) was higher than that reported in previous studies (49.5\%)[11]. The correlation between the length of the septal perforator artery on CCTA and the length of LLGE on LGE was also more significant, indicating that the two were close in length. This may be explained by the following: 1) The acquisition sequence of the LGE images in this study was completed through the phase-sensitive inversion recovery technique (PSIR), which has a high signal-tonoise ratio compared to conventional Scout $\mathrm{TI}$, and an enhanced area can be clearly displayed on LGE images[13], with a higher detection rate for the LLGE in the anterior septum; 2) The anterior septal perforator arteries originate from the proximal part of the left anterior descending branch (LAD) of the coronary artery and supply the upper two-thirds part of the interventricular septum, with their number varying from 4 to 13 and the first one being the thickest and longest[14]. With the advantage of the high contrast of the PSIR technique, these small vessels were shown on LGE. As shown in Fig. 6, two parallel LLGE in the anterior septum can be clearly observed. In addition, the length of the anterior septal perforator artery on CCTA in patients with LLGE + was significantly longer than that in patients with LLGE-. Based on this analysis, the LLGE in the anterior septum is most likely to represent an anterior septal perforator artery and more than one will be visualized on LGE.

In the present study, an LLGE similar to that of the anterior septum appeared in the lateral wall on LGE among 10 patients $(10 / 58,17.2 \%)$, with more obvious enhancement. LLGE in the lateral wall was visually confirmed as the circumflex branch artery on CCTA. The circumflex branch artery originates from the left coronary artery, most of which is on the surface of the myocardium, but some such arteries travel inside the myocardium and are surrounded by it, forming what is called the "myocardial bridge." The likelihood of the myocardial bridge occurring in the circumflex branch artery is lower[15-17], which may also explain why the probability of LLGE in the lateral wall is much lower compared to that of the anterior septum. The lateral wall of the left ventricular myocardium includes not only the circumflex branch arteries, but also their branches, veins, capillaries, and sinusoids[18], which may all exhibit LLGE, as well. LLGE in the lateral wall was considered to represent the circumflex branch artery for the following reasons: (1) The LLGE located in the lateral wall is highly correlated in length with the circumflex branch 
arteries on the CCTA; (2) In the lateral wall of the short-axis views of the left ventricle on CCTA, the circumflex branch artery originated from the left coronary artery and passed through the myocardium, and in analyzing the LGE images layer by layer from the base to the apical part, the origin and shape of the LLGE was similar to the corresponding circumflex artery, as shown in Fig. 4; and (3) The PSIR is a gradient echo sequence with a short TR time, which has an inflow-enhancement effect for the fast intraarterial flow, and the LGE images are acquired during the diastolic phase, which is beneficial for arterial visualization. The circumflex branch artery could be seen in 4 patients $(4 / 58,6 \%)$ on CCTA, but there was no LLGE in the lateral wall, probably due to the higher spatial resolution of the CCTA rather than that of the LGE. Therefore, the LLGE appearing in the lateral wall may represent the circumflex branch artery that travels within the myocardium.

Myocardial fibrosis is a common pathological feature of HCM. Studies have shown that the prediction of cardiac adverse events by quantitative LGE is superior to the criteria recommended by current guidelines, so it is clinically important to determine the presence and extent of LGE in patients with HCM[19-22]. However, in the present study, the presence of LLGE led to an overestimation of the degree of myocardial fibrosis among patients with HCM. The LGE\% decreased significantly after the removal of LLGE, which was more obvious under multiple LLGE, as shown in Fig. 5, where LGE\% decreased from 14.8 to $4.9 \%$; this is highly unfavorable for regular follow-up and evaluation of the condition of HCM patients. In addition, the presence of LLGE can also be confused with the enhancement of a focal lesion, as seen in Fig. 4A, and without the comparison of CCTA, the patient is more likely to be considered to have a history of myocarditis.

Through observation, the arteries seen on LGE had the following characteristics: (1) LLGE mostly travels against the myocardial epicardium, with only a few vessels travelling deep inside the myocardium; (2) By tracking the LGE images upward, the starting point of LLGE may originate from its superior vessels, but sometimes the focus lesion will affect how it is seen, such as in myocardial amyloidosis. Radiologists should identify LLGE based on vascular anatomy and clinical history so as to avoid misdiagnosis.

This study had several limitations. The overall number of subjects was relatively small, so there may have been some bias in the analysis of LLGE localization or comparison of the results of two LGE\% measurements before and after removal of LLGE among patients with HCM. In addition, CCTA is not the gold standard for identifying vessels, but rather coronary angiography in process. However, under existing conditions, CCTA is also representative of some of the vessels, and future prospective studies based on coronary angiography are needed to confirm the true origin of LLGE.

\section{Conclusions}

The LLGE appearing in the anterior septum and lateral wall may not represent an enhancement area of the lesion, but rather an anterior septal perforator artery and circumflex artery. This LLGE overestimates the extent of myocardial fibrosis in patients with HCM. When encountering LLGE in the anterior septum 
and lateral wall, careful analysis is required to determine whether it is a lesion or simply contrast enhancement of the vessel.

\section{Declarations}

Author contribution:All authors contributed to the study conception and design and all authors commented on previous versions of the manuscript. All authors read and approved the final manuscript.

Funding: This work was funded by the National Natural Science Foundation of China (grant number 81971587) and the Natural Science Foundation of Chongqing (grant number cstc2020jcyj-msxmX0399)

Ethics approval: The study was conducted according to the guidelines of the Declaration of Helsinki and approved by Ethics Committee of the First Affiliated Hospital of Army Medical University, PLA(KY2020314).

Informed Consent Statement: The study was designed as a retrospective chart review, so written patient consent was waived.

Data Availability Statement: The data that support the findings of this study are available from the corresponding author upon request.

Conflicts of interest statement: The authors have no potential conflicts of interest.

Acknowlsedgments: Not applicable.

Conflicts of interest statement and funding: The authors have no potential conflicts of interest.

\section{References}

1. Kidoh M, Oda S, Utsunomiya D, Emoto T, Nakaura T, Nagayama Y et al. Basal septal perforator vein mimicking the "late iodine enhancement" in delayed phase cardiac CT for myocardial scar assessment. Radiol Case Rep 2019;14:588-590. https://doi.org/ 10.1016/j.radcr.2019.02.016

2. Mahrholdt H, Goedecke C, Wagner A, Meinhardt G, Athanasiadis A, Vogelsberg H et al. Cardiovascular magnetic resonance assessment of human myocarditis: A comparison to histology and molecular pathology. CIRCULATION 2004;109:1250-1258. https://doi.org/

10.1161/01.CIR.0000118493.13323.81

3. Assomull RG, Prasad SK, Lyne J, Smith G, Burman ED, Khan M et al. Cardiovascular magnetic resonance, fibrosis, and prognosis in dilated cardiomyopathy. J AM COLL CARDIOL 2006;48:19771985. https://doi.org/ 10.1016/j.jacc.2006.07.049

4. Viallon M, Jacquier A, Rotaru C, Delattre BM, Mewton N, Vincent F et al. Head-to-head comparison of eight late gadolinium-enhanced cardiac MR (LGE CMR) sequences at 1.5 tesla: From bench to bedside. J MAGN RESON IMAGING 2011;34:1374-1387. https://doi.org/ 10.1002/jmri.22783 
5. Flett AS, Hasleton J, Cook C, Hausenloy D, Quarta G, Ariti C et al. Evaluation of techniques for the quantification of myocardial scar of differing etiology using cardiac magnetic resonance. JACC Cardiovasc Imaging 2011;4:150-156. https://doi.org/ 10.1016/j.jcmg.2010.11.015

6. Mahrholdt H, Wagner A, Judd RM, Sechtem U, Kim RJ. Delayed enhancement cardiovascular magnetic resonance assessment of non-ischaemic cardiomyopathies. EUR HEART J 2005;26:14611474. https://doi.org/ 10.1093/eurheartj/ehi258

7. Ferreira PF, Gatehouse PD, Mohiaddin RH, Firmin DN. Cardiovascular magnetic resonance artefacts. J Cardiovasc Magn Reson 2013;15:41. https://doi.org/ 10.1186/1532-429X-15-41

8. Kellman P, Arai AE, McVeigh ER, Aletras AH. Phase-sensitive inversion recovery for detecting myocardial infarction using gadolinium-delayed hyperenhancement. MAGN RESON MED 2002;47:372-383. https://doi.org/ 10.1002/mrm.10051

9. Halliday BP, Baksi AJ, Gulati A, Ali A, Newsome S, Izgi C et al. Outcome in dilated cardiomyopathy related to the extent, location, and pattern of late gadolinium enhancement. JACC Cardiovasc Imaging 2019;12:1645-1655. https://doi.org/ 10.1016/j.jcmg.2018.07.015

10. Cheng S, Fang M, Cui C, Chen X, Yin G, Prasad SK et al. LGE-CMR-derived texture features reflect poor prognosis in hypertrophic cardiomyopathy patients with systolic dysfunction: Preliminary results. EUR RADIOL 2018;28:4615-4624. http://doi.org/ 10.1007/s00330-018-5391-5

11. Nakamura M, Kido T, Hirai K, Tabo K, Tanabe $\mathrm{Y}$, Kawaguchi $\mathrm{N}$ et al. What is the mid-wall linear high intensity "lesion" on cardiovascular magnetic resonance late gadolinium enhancement? J Cardiovasc Magn Reson 2020;22:66. http://doi.org/ 10.1186/s12968-020-00665-5

12. Moravsky G, Ofek E, Rakowski H, Butany J, Williams L, Ralph-Edwards A et al. Myocardial fibrosis in hypertrophic cardiomyopathy: Accurate reflection of histopathological findings by CMR. JACC Cardiovasc Imaging 2013;6:587-596. http://doi.org/ 10.1016/j.jcmg.2012.09.018

13. Varga-Szemes A, van der Geest RJ, Spottiswoode BS, Suranyi P, Ruzsics B, De Cecco CN et al. Myocardial late gadolinium enhancement: Accuracy of T1 mapping-based synthetic InversionRecovery imaging. RADIOLOGY 2016;278:374-382. http://doi.org/ 10.1148/radiol.2015150162

14. Topaz O, DiSciascio G, Vetrovec GW. Septal perforator arteries: From angiographic-morphologic characteristics to related revascularization options. AM HEART J 1992;124:810-815. http://doi.org/ 10.1016/0002-8703(92)90304-e

15. O'Brien JP, Srichai MB, Hecht EM, Kim DC, Jacobs JE. Anatomy of the heart at multidetector CT: What the radiologist needs to know. RADIOGRAPHICS 2007;27:1569-1582. http://doi.org/ $10.1148 /$ rg. 276065747

16. Corban MT, Hung OY, Eshtehardi P, Rasoul-Arzrumly E, McDaniel M, Mekonnen G et al. Myocardial bridging: Contemporary understanding of pathophysiology with implications for diagnostic and therapeutic strategies. J AM COLL CARDIOL 2014;63:2346-2355. http://doi.org/ 10.1016/j.jacc.2014.01.049

17. Hsieh PJ, Su HY, Lo HS, Chen ML. Dipyridamole 201TI myocardial SPECT in the assessment of a patient with myocardial bridging and concomitant atherosclerotic coronary artery disease. CLIN 
NUCL MED 2012;37:e257-e262. http://doi.org/ 10.1097/RLU.0b013e318244429f

18. Saremi F, Muresian H, Sánchez-Quintana D. Coronary veins: Comprehensive CT-anatomic classification and review of variants and clinical implications. RADIOGRAPHICS 2012;32:E1-E32. http://doi.org/ 10.1148/rg.321115014

19. Freitas $P$, Ferreira AM, Arteaga-Fernández E, de Oliveira AM, Mesquita J, Abecasis $\mathrm{J}$ et al. The amount of late gadolinium enhancement outperforms current guideline-recommended criteria in the identification of patients with hypertrophic cardiomyopathy at risk of sudden cardiac death. $\mathrm{J}$ Cardiovasc Magn Reson 2019;21:50. http://doi.org/ 10.1186/s12968-019-0561-4

20. Axelsson RA, Farhad H, Valente AM, Couce JP, Jefferies JL, Bundgaard H et al. Prevalence and progression of late gadolinium enhancement in children and adolescents with hypertrophic cardiomyopathy. CIRCULATION 2018;138:782-792. http://doi.org/

10.1161/CIRCULATIONAHA.117.032966

21. van der Velde N, Hassing HC, Bakker BJ, Wielopolski PA, Lebel RM, Janich MA et al. Improvement of late gadolinium enhancement image quality using a deep learning-based reconstruction algorithm and its influence on myocardial scar quantification. EUR RADIOL 2020. http://doi.org/ 10.1007/s00330-020-07461-w

22. Patel AR, Kramer CM. Role of cardiac magnetic resonance in the diagnosis and prognosis of nonischemic cardiomyopathy. JACC Cardiovasc Imaging 2017;10:1180-1193. http://doi.org/ 10.1016/j.jcmg.2017.08.005

\section{Tables}


Table 1

Clinical characteristics of patients with LLGE + and LLGE-

\begin{tabular}{|llll|}
\hline Characteristics & LLGE+ & LLGE- & p value \\
\hline $\begin{array}{l}\text { Number of } \\
\text { patients }\end{array}$ & 40 & 18 & \\
\hline Age (years) & $45 \pm 15$ & $45 \pm 17$ & 0.894 \\
\hline Male & $34(85.0 \%)$ & $13(72.2 \%)$ & 0.250 \\
\hline Diabetes & $5(12.5 \%)$ & $2(11.1 \%)$ & 0.880 \\
\hline Hypertension & $4(10.0 \%)$ & $16(88.8 \%)$ & $<0.001$ \\
\hline CAD & $9(22.5 \%)$ & $6(33.3 \%)$ & 0.759 \\
\hline $\begin{array}{l}\text { Heart rate } \\
\text { (beats/min) }\end{array}$ & $65 \pm 5$ & $66 \pm 5$ & 0.325 \\
\hline LVEF $(\%)$ & $58 \pm 9$ & $57 \pm 12$ & 0.590 \\
\hline LVEDV $(\mathrm{ml})$ & $133 \pm 34$ & $134 \pm 55$ & 0.901 \\
\hline LVESV $(\mathrm{ml})$ & $56 \pm 26$ & $66 \pm 48$ & 0.888 \\
\hline DCM & $1(2.5 \%)$ & $2(11.1 \%)$ & 0.224 \\
\hline HCM & $7(17.5 \%)$ & $6(33.3 \%)$ & 0.181 \\
\hline Other & $8(20.0 \%)$ & $5(27.7 \%)$ & 0.511 \\
\hline
\end{tabular}

LLGE = linear late gadolinium enhancement; CAD = coronary artery disease; LVEF = left ventricular ejection fraction; LVEDV = left ventricular end-diastolic volume; LVESV = left ventricular end-systolic volume; $\mathrm{DCM}=$ dilated cardiomyopathy; $\mathrm{HCM}$ = hypertrophic cardiomyopathy

Table 2

Number of patients with LLGE in the anterior septum and anterior septal perforator artery on

CCTA

\begin{tabular}{|llll|}
\hline & CCTA+ & CCTA- & Total \\
\hline LLGE+ & 35 & 0 & 35 \\
LLGE- & 19 & 4 & 23 \\
Total & 54 & 4 & 58 \\
\hline
\end{tabular}

LLGE = linear late gadolinium enhancement; CCTA+ = patient in whom an anterior septal perforator artery could be identified on coronary CTA; CCTA- = patient in whom an anterior septal perforator artery could not be identified on coronary CTA 
Table 3

Number of patients with the LLGE location

\begin{tabular}{|llll|}
\hline & $\begin{array}{l}\text { Anterior } \\
\text { septum }\end{array}$ & $\begin{array}{l}\text { Lateral } \\
\text { wall }\end{array}$ & Total \\
\hline Sub-epicardium & 27 & 8 & 35 \\
\hline Intramyocardium & 8 & 2 & 10 \\
\hline Total & 35 & 10 & 45 \\
\hline
\end{tabular}

Table 4

Number of patients with LLGE in lateral wall and circumflex artery on CCTA

\begin{tabular}{|llll|}
\hline & CCTA+ & CCTA- & Total \\
\hline LLGE+ & 10 & 0 & 10 \\
\hline LLGE- & 4 & 44 & 48 \\
\hline Total & 14 & 44 & 58 \\
\hline
\end{tabular}

LLGE = linear late gadolinium enhancement, CCTA+ = patient in which a circumflex artery could be identified on coronary CTA, CCTA- = patient in which a circumflex artery could not be identified on coronary CTA

Figures 

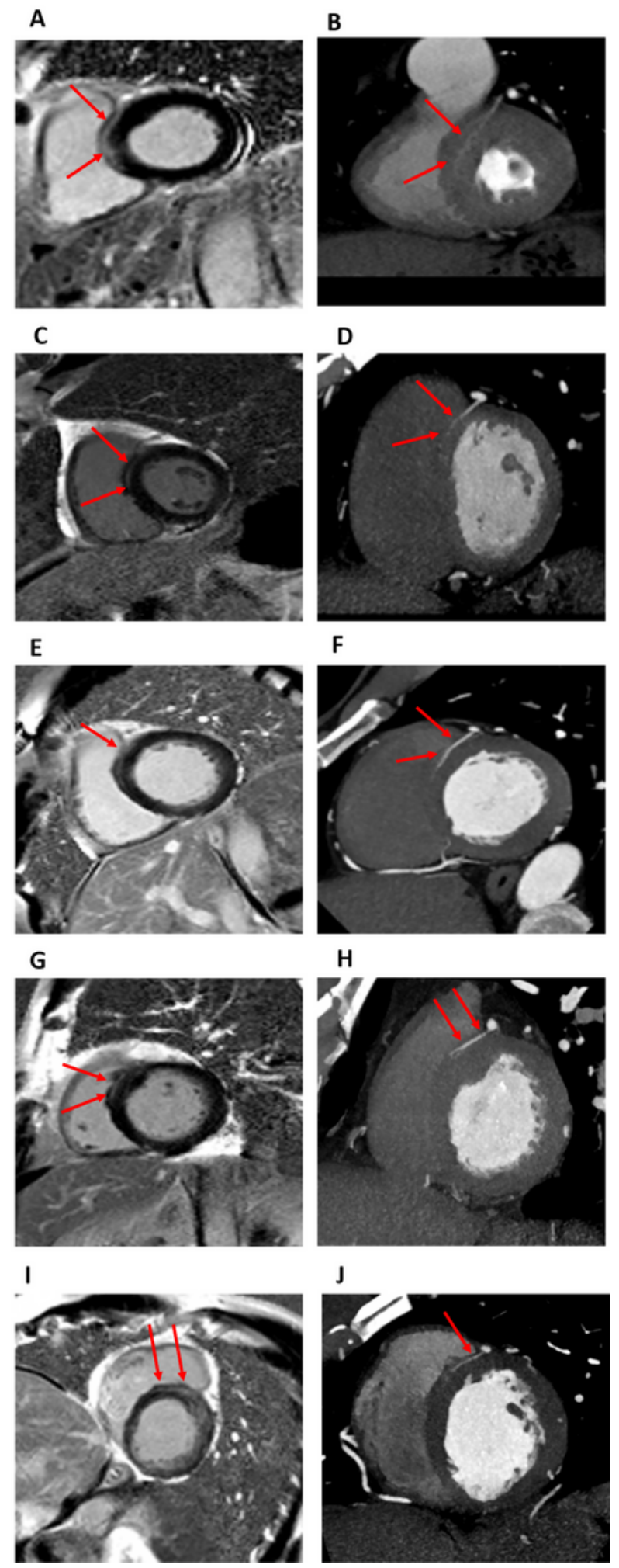

Figure 1

Some patients with LLGE and anterior septal perforator arteries on CCTA. a-b. An 87-year-old male with myocardial infarction in the lateral wall. a. LLGE on CMR, b. anterior septal perforator arteries on CCTA. Cd. A 51-year-old male. c. LLGE on CMR, d. anterior septal perforator arteries on CCTA. e-f. A 43-year-old male. e LLGE on CMR, f. anterior septal perforator arteries on CCTA. g-h A 54-year- old male with hypertrophic cardiomyopathy. g. LLGE on CMR, h. anterior septal perforator arteries on CCTA. i-j. A 31- 
year-old with hypertrophic cardiomyopathy. i. LLGE on CMR, j. anterior septal perforator arteries on CCTA. LLGE = linear late gadolinium enhancement, CCTA = coronary computed tomography angiography, CMR $=$ cardiac magnetic resonance

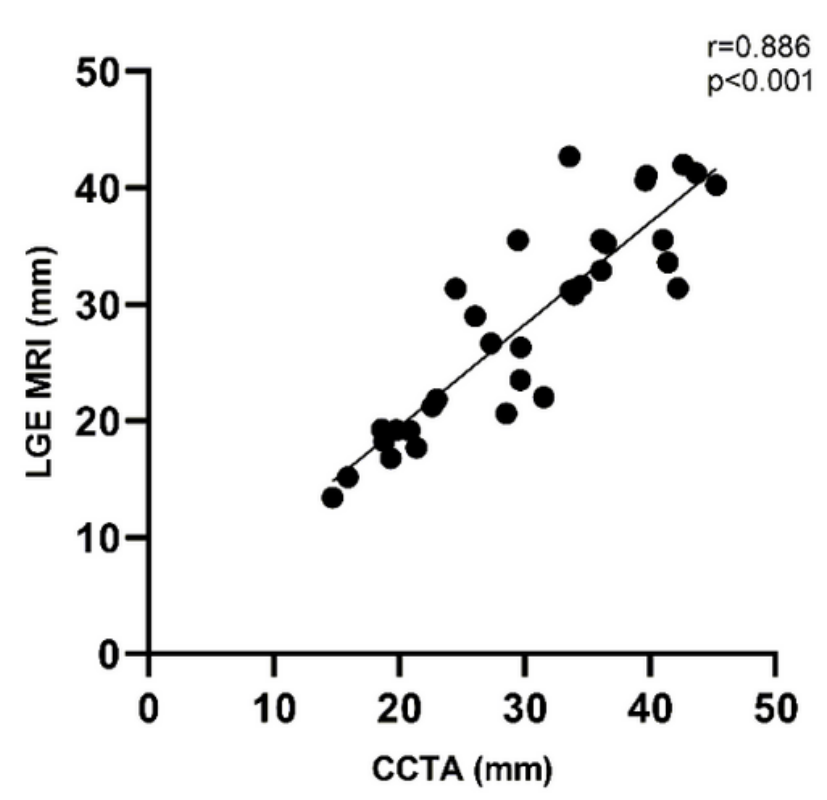

(a)

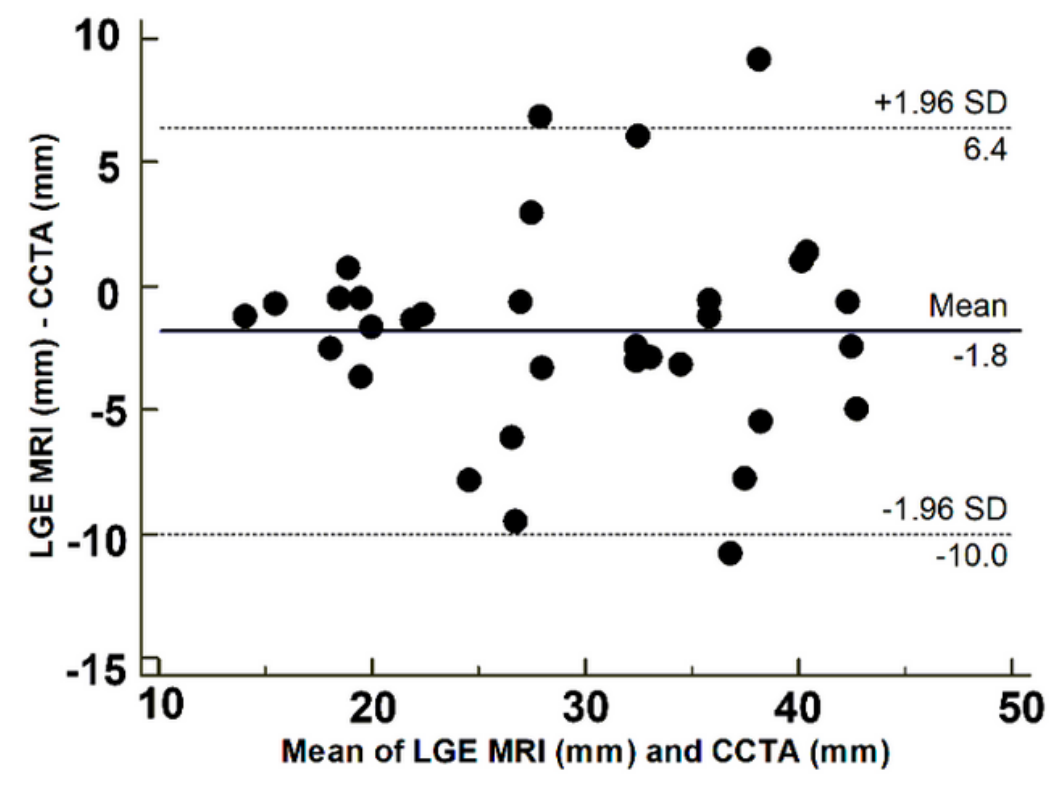

(b)

Figure 2

Correlation between LLGE length measurements on CMR and length of anterior septal perforator arteries on CCTA. There was a strong correlation between LLGE length measurements on CMR and the length of the anterior septal perforator arteries on CCTA. LLGE = linear late gadolinium enhancement, CMR = cardiac magnetic resonance, CCTA = coronary computed tomography angiography 


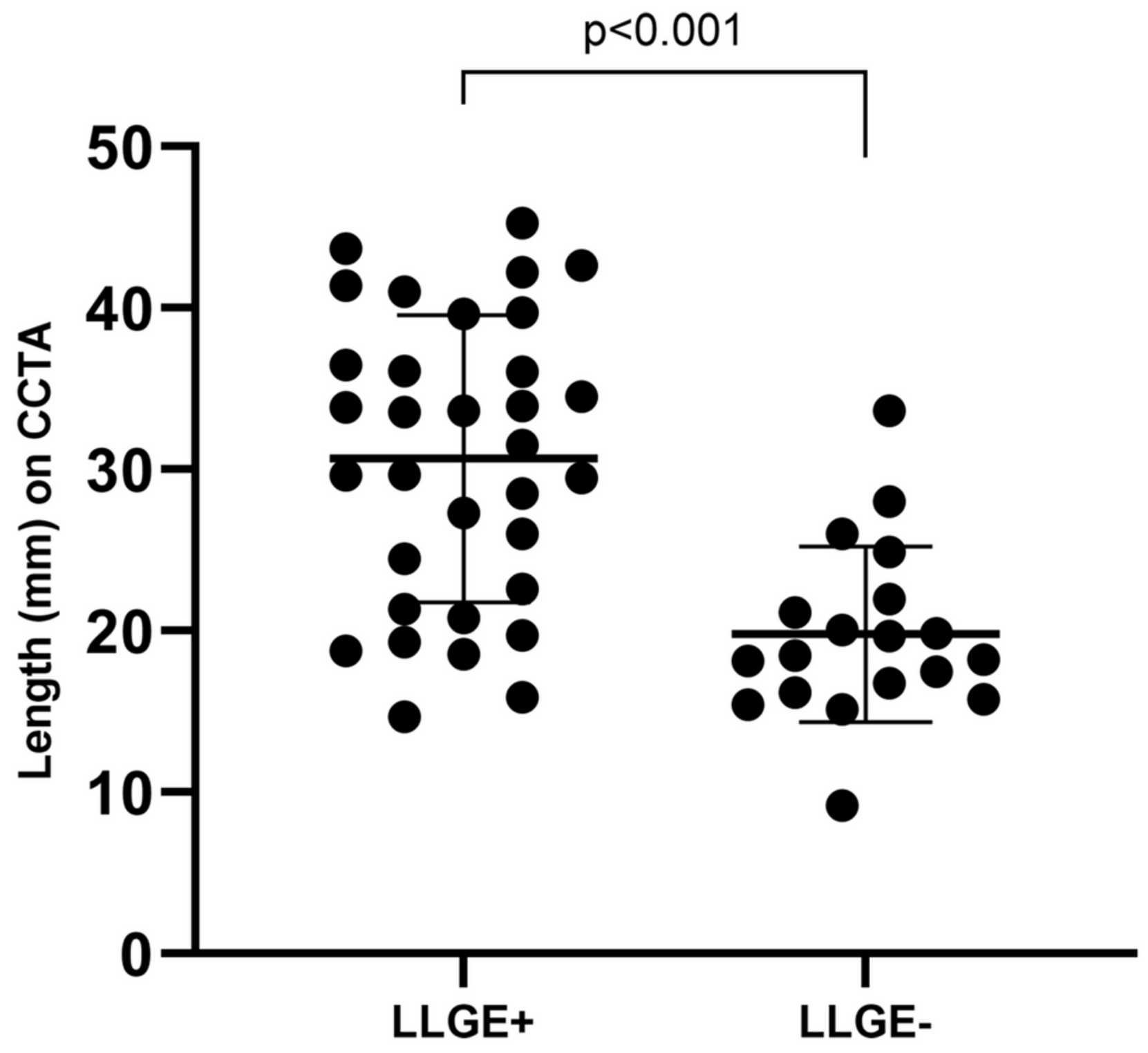

Figure 3

Comparison between LLGE+ and LLGE- patients in the measured lengths of anterior septal perforator arteries by CCTA. LLGE = linear late gadolinium enhancement, CCTA = coronary computed tomography angiography 

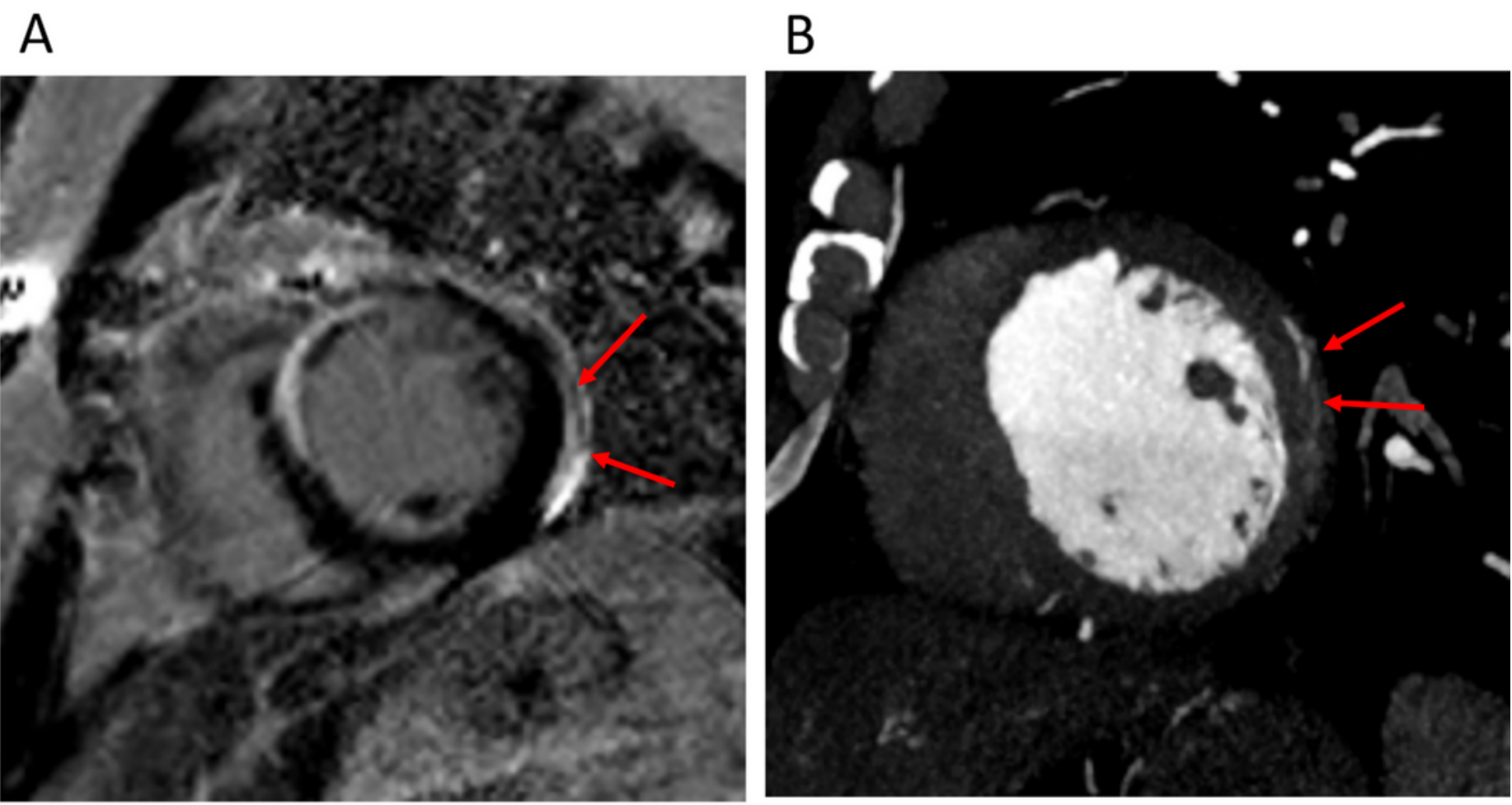

C

D
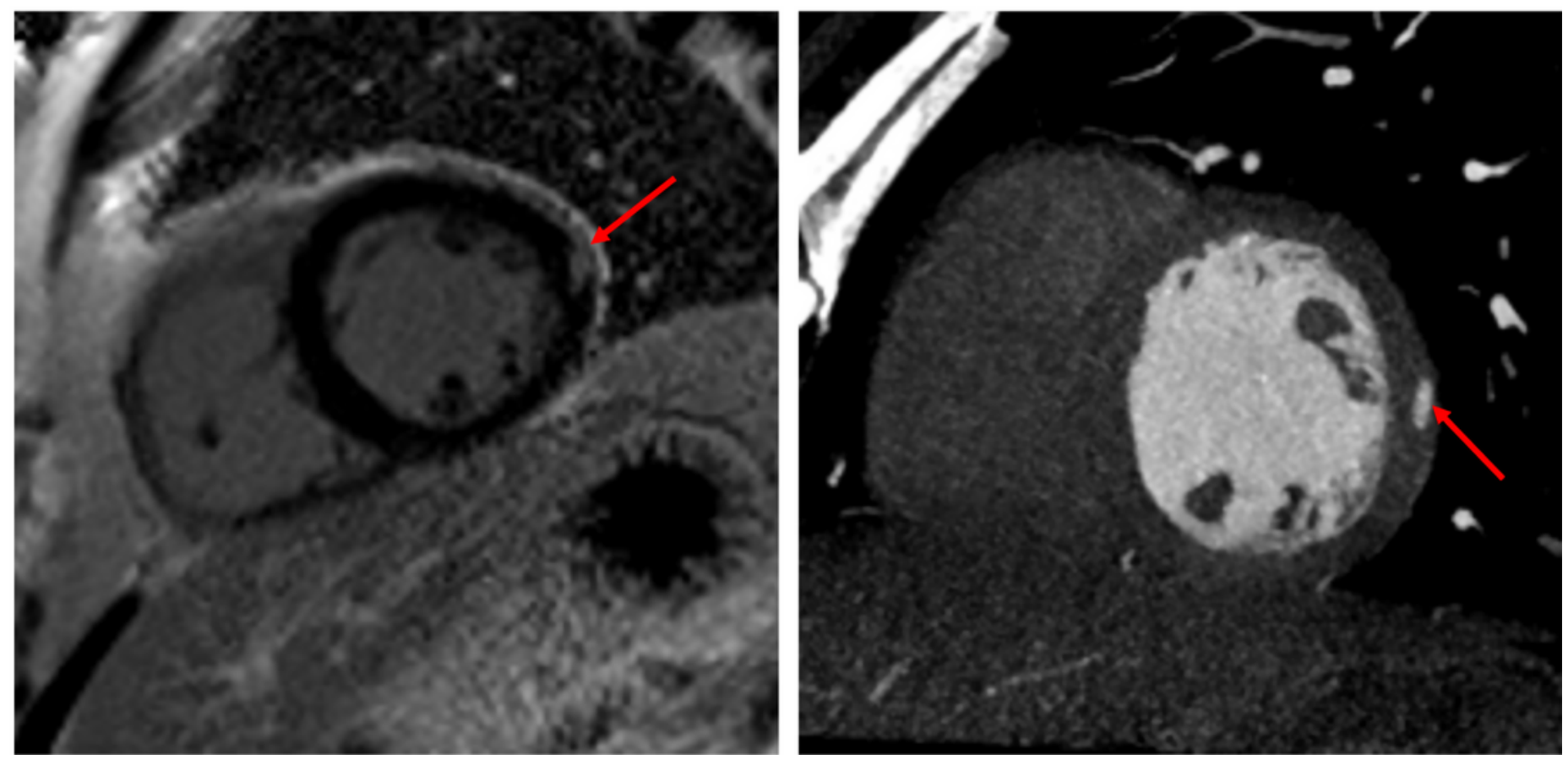

Figure 4

Some patients with LLGE and circumflex arteries on coronary computed tomography angiography (CCTA). a-b. A 66-year-old male with an old myocardial infarction in the anterior septum. a. LLGE on CMR, b. circumflex arteries on CCTA. c-d. A 46-year- old male. c. LLGE on CMR, d. circumflex arteries on CCTA. LLGE = linear late gadolinium enhancement, CCTA = coronary computed tomography angiography , CMR $=$ cardiac magnetic resonance 


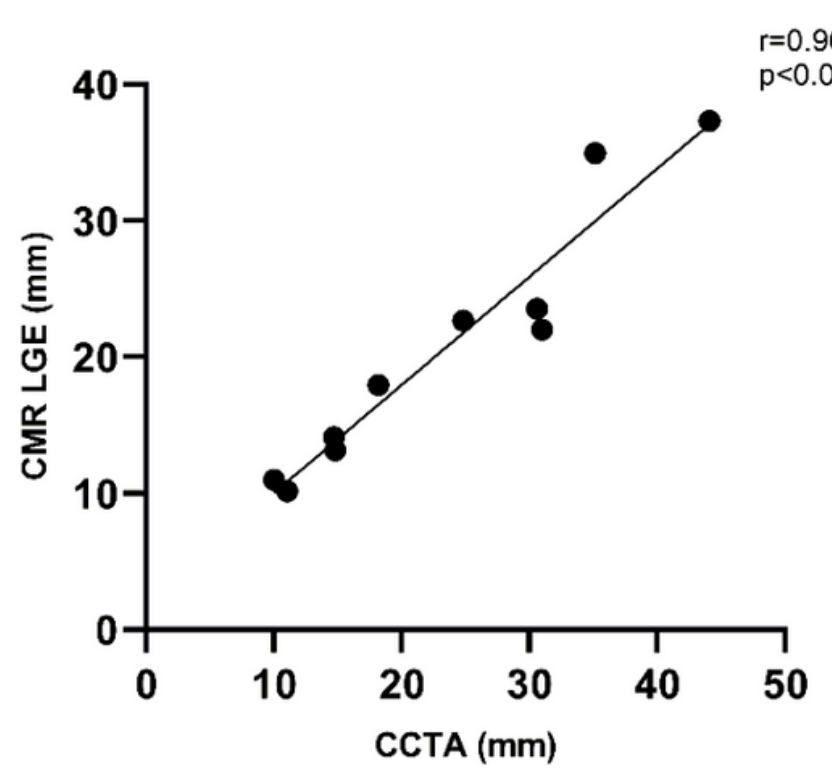

(a)

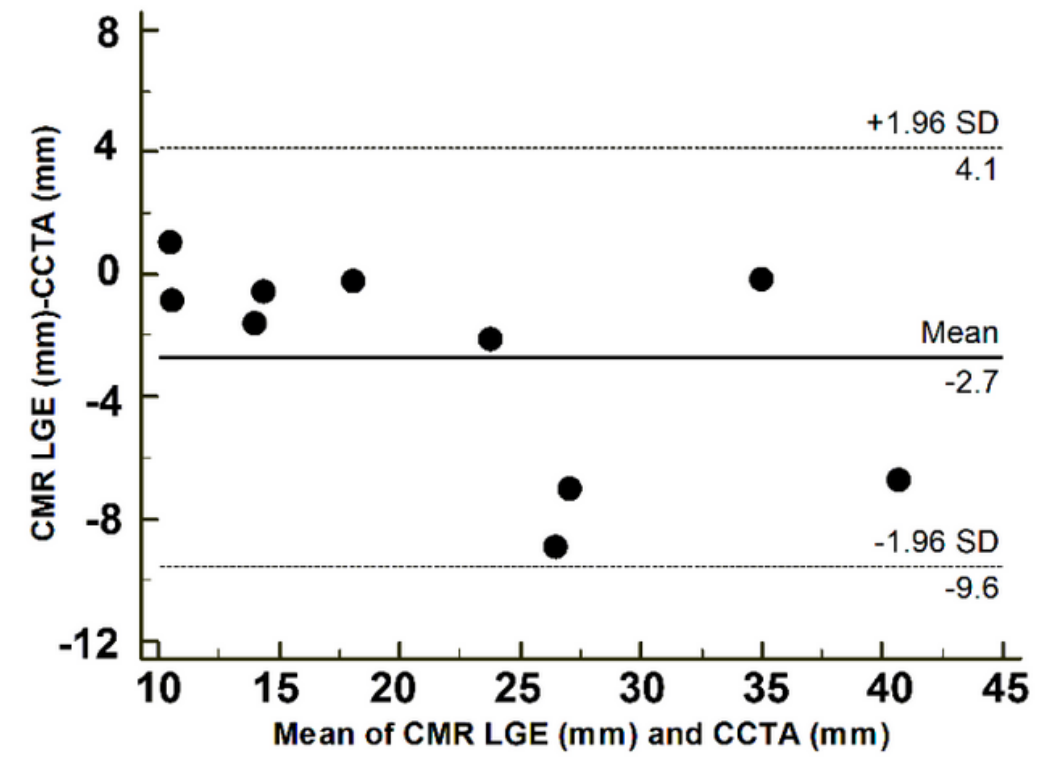

(b)

Figure 5

Correlation between LLGE length measurements on CMR and the length of the circumflex branch arteries on CCTA. There was a strong correlation between the LHI length measurements on CMR and the length of the circumflex branch arteries on CCTA. LLGE = linear late gadolinium enhancement, CMR = cardiac magnetic resonance, CCTA = coronary computed tomography angiography 

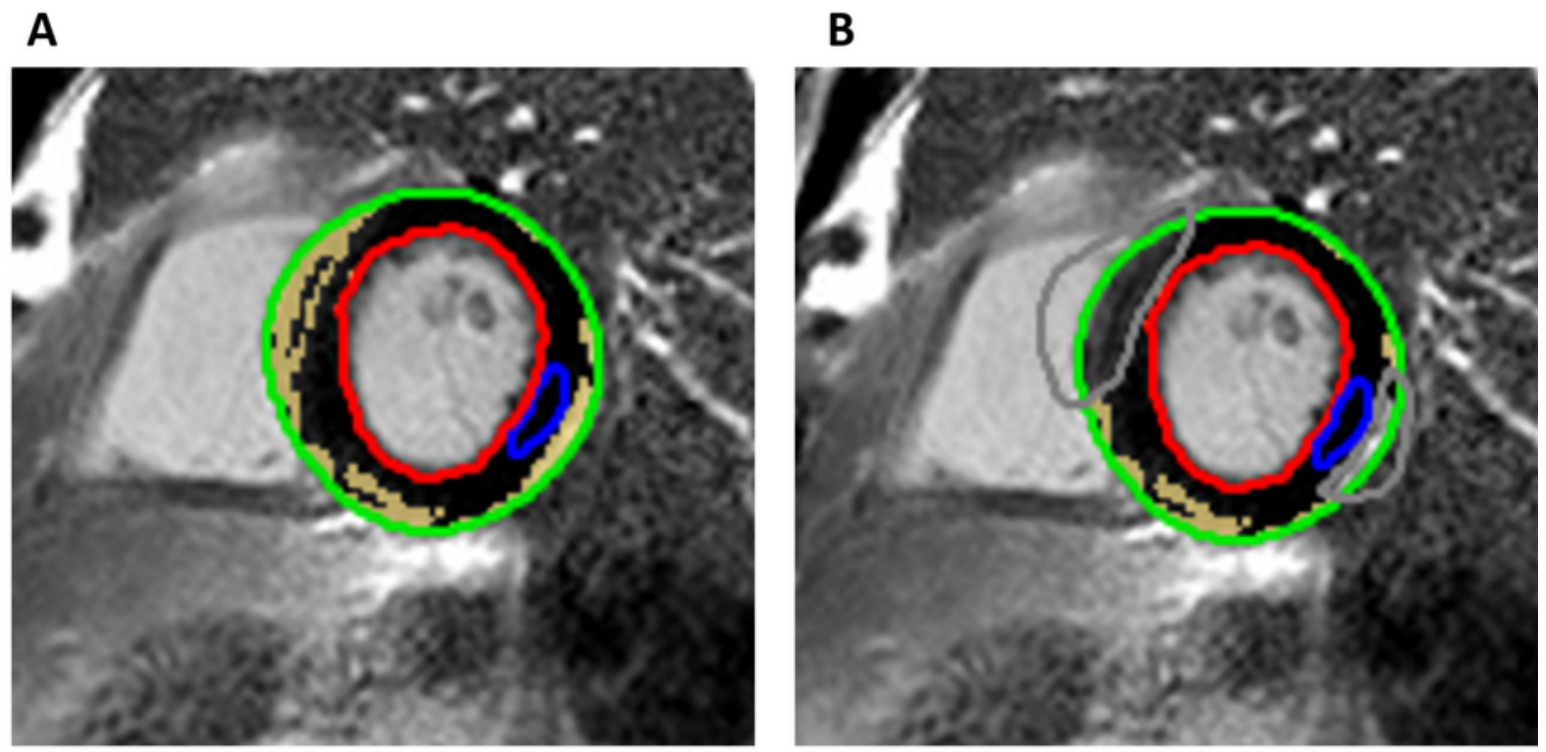

C

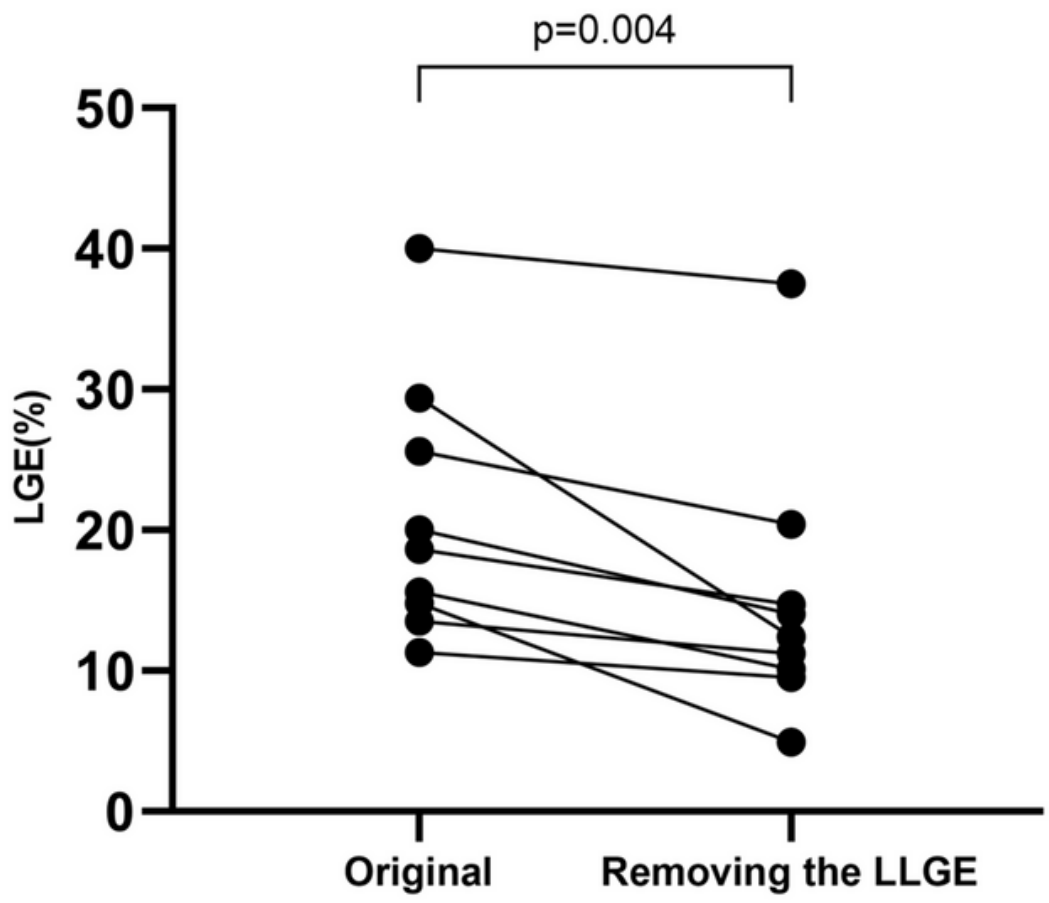

Figure 6

An HCM patient with two anterior septal perforator arteries in the anterior septum and circumflex branch artery in the lateral wall observed on LGE. a. Original measurement of LGE\%, b. Second measurement of LGE\% after removing the LLGE manually. c. Comparison of the LGE\% between the two measurements among the HCM patients. After removing the LLGE, the LGE\% was significantly reduced $(p=0.004)$. $\mathrm{HCM}=$ hypertrophic cardiomyopathy, LLGE = linear late gadolinium enhancement 


\section{Supplementary Files}

This is a list of supplementary files associated with this preprint. Click to download.

- SupplementaryFig.1.docx 